\title{
Interview
}

\section{Die Bill Clinton-Regel}

\author{
CallCenterProfi traf Carsten K. Rath, Grand Hotelier und Unternehmer, \\ und sprach mit ihm bereits vorab über seinen Vortrag, den er auf der \\ CCW 2013 in Berlin halten wird.
}

CallCenterProfi: Herr Rath, für Unternehmen zählen auf dem Weg zum Erfolg laut Ihrer These vor allem die ersten 5 Sekunden. Warum gerade 5 Sekunden?

Rath: Nicht nur die 5 Sekunden, jedes Mal wenn wir auf Menschen treffen - Kunden, Gäste, Mitarbeiter - dann entscheiden wir ob wir den Moment achtsam und besonders gestalten - also nur bei unserem Gegenüber sind oder wertlos, weil achtlos dem Menschen gegenüber und somit diesen vorüberziehen lassen. Menschen binden Menschen an Marken und Produkte durch ihre Dienstleistung, und wenn laut Kopper „alle Mitarbeiter jeden Kunden, den sie sehen, freundlich begrüßen würden, würden wir unseren Umsatz im Inland um 25 Prozent steigern".

CallCenterProfi: Was bedeutet in diesem Zusammenhang genau der Bill ClintonFactor?

Rath: Bill Clinton dankte meinen Abteilungsleitern und mir einmal nach einem Staatsbankett im Adlon, Berlin. Dabei lebte er diesen Augenblick perfekt. Nichts und niemand, keine Sicherheitsleute, keine Protokolle, nicht einmal die ihm wirklich

\section{TIPP}

\section{Vortrag live erleben!}

Mehr zum „The Bill Clinton-Factor - die 5-SekundenRegel" erfahren Sie von Carsten K. Rath auf der CCW.

Tag: Mittwoch 27. Februar 2013

Beginn: $10.15 \mathrm{Uhr}$

Der zweite Kongresstag steht übrigens ganz im Zeichen der Kundenbindung - „Ist der Kunde bereit, für mehr Service zu zahlen?". näher bekannte Praktikantin Monica Lewinsky konnte ihn in diesem Moment des Dankes ablenken. Es waren nur er und wir - seine Gegenüber - 5 Sekunden der Wertschätzung, 5 Sekunden ungeteilte Aufmerksamkeit, 5 Sekunden Achtung. Daraus ist der Bill Clinton-Factor entstanden.

CallCenterProfi: Wie würden Sie die Rolle der Mitarbeiter beschreiben? Welche besonderen Herausforderungen sehen Sie hier? Rath: In jedem Unternehmen sind wir Mitarbeiter Dienstleister, also wir dienen durch unsere Leistung. Damit meine ich nichts Devotes, aber etwas Präzises, Herzliches und Schönes. Fachwissen kann man sich aneignen, Herzlichkeit und Empathie jedoch nicht. Und um erfolgreich in der Dienstleistung zu sein, gehört Empathie, überdurchschnittlich viel Empathie dazu, um seine Kunden zu begeistern und sie somit an das Unternehmen zu binden.

CallCenterProfi: Und der Kunde. Wie bekommt man als Unternehmen einen treuen und zufriedenen Kunden? Rath: Jeder Mensch will ernst genommen werden - verstanden werden. Einer der wichtigsten und erfolgreichsten Wege sind die „3 goldenen Regeln“.

Ich begrüße jeden Kunden persönlich mit einem ehrlichen Lächeln. Ich stelle mich dem Kunden mit meinem Namen vor und erfahre so den Namen des Kunden.

Diskret halte ich meine Augen und Ohren offen, um die Wünsche meiner Kunden wahrzunehmen und zu erfüllen. Somit übertreffe ich ihre Erwartungen. Ich verabschiede jeden Kunden persönlich mit einem herzlichen Dank und verbinde dies mit dem ehrlichen Wunsch nach einem baldigen Wiedersehen. Wenn wir diese 3 Regeln befolgen und

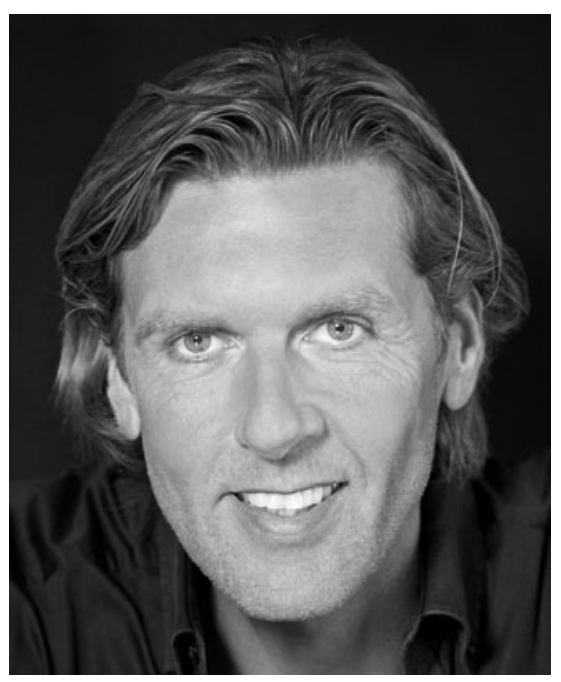

dabei professionell und herzlich bei der Sache sind, können wir jeden Kunden begeistern und ihn von unserem Produkt oder Service begeistern. Die Neukundenakquise ist um einiges teurer, als einen unzufriedenen Kunden wieder von seinem Unternehmen zu überzeugen. Letzteres bedarf der eben angesprochenen Empathie, wenn die nicht vorhanden ist, nützt auch der beste Rückgewinnungsprozess nichts.

CallCenterProfi: Sie sind auch Kunde. Welche Erlebnisse hatten Sie persönlich schon mit Call Centern? Gerne die besonders guten, aber auch die schlechten... Rath: Diesbezüglich habe ich schon beides erlebt. Zu den Erlebnissen empfehle ich Ihnen einen Link auf YouTube - eine Art persönliche Erfahrung, wenn Sie so wollen. Dieser trifft es bestens. $\leftarrow$

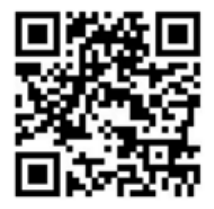

